\title{
FLAVOR OSCILLATIONS OF LOW ENERGY NEUTRINOS IN THE ROTATING NEUTRON STAR
}

\author{
Maxim Dvornikov ${ }^{a}$ \\ Departamento de Fúsica y Centro de Estudios Subatómicos, \\ Universidad Técnica Federico Santa María, Casilla 110-V, Valparaíso, Chile and \\ IZMIRAN, 142190, Troitsk, Moscow region, Russia \\ Abstract.We study flavor oscillations of low energy neutrinos propagating in dense \\ matter of a rotating neutron star. On the basis of the exact solutions of the wave \\ equations for neutrinos mass eigenstates we derive the transition probability for \\ neutrinos having big initial angular momentum. It is found that flavor oscillations \\ of neutrinos with energies of several electron-Volts can be resonancely enhanced.
}

It is known that neutrinos play a significant role at the last stages of the evolution of massive stars. For example, almost $99 \%$ of the gravitational energy of a protoneutron star is carried away during the supernova explosion. The remaining dense, compact object, a neutron star, can have extreme properties: central density $\sim 10^{14} \mathrm{~g} / \mathrm{cc}$, magnetic field $\sim 10^{15} \mathrm{G}$ and angular velocity $\sim$ $10^{3} \mathrm{~s}^{-1}$. In this short note we examine the influence of the neutron star rotation on flavor oscillations of neutrinos. Note that the propagation of neutrinos in rotating matter was also studied in Refs. [1,2].

Let us study the evolution of the two flavor neutrinos system $\left(\nu_{\alpha}, \nu_{\beta}\right)$ interacting with the background matter by means of the electroweak forces. The Lagrangian for this system has the form,

$$
\mathcal{L}=\sum_{\lambda=\alpha, \beta} \bar{\nu}_{\lambda}\left(\mathrm{i} \gamma^{\mu} \partial_{\mu}-f_{\lambda}^{\mu} \gamma_{\mu}^{\mathrm{L}}\right) \nu_{\lambda}-\sum_{\lambda \lambda^{\prime}=\alpha, \beta} m_{\lambda \lambda^{\prime}} \bar{\nu}_{\lambda} \nu_{\lambda^{\prime}}
$$

where $\gamma_{\mu}^{\mathrm{L}}=\gamma_{\mu}\left(1-\gamma^{5}\right) / 2$. Supposing that matter is electroneutral and all the background fermions rotate as a rigid body we can express the external fields $f_{\lambda}^{\mu}$ for $\alpha=\mu$ or $\tau$ and $\beta=e$ oscillations channel as,

$$
f_{\alpha}^{\mu}=-\frac{G_{\mathrm{F}}}{\sqrt{2}} j_{n}^{\mu}, \quad f_{\beta}^{\mu}=\frac{G_{\mathrm{F}}}{\sqrt{2}}\left(2 j_{e}^{\mu}-j_{n}^{\mu}\right), \quad j_{e, n}^{\mu}=\left(n_{e, n}, n_{e, n} \mathbf{v}\right),
$$

where $G_{\mathrm{F}}$ is the Fermi constant, $n_{e, n}$ is the number density of electrons and neutrons and $\mathbf{v}=(\boldsymbol{\Omega} \times \mathbf{r})$ is the velocity of the background matter.

To study the evolution of the system (1) we should introduce the neutrino mass eigenstates $\psi_{a}$ to diagonalize the mass matrix $\left(m_{\lambda \lambda^{\prime}}\right), \nu_{\lambda}=\left(\exp \left[-\mathrm{i} \sigma_{2} \theta\right]\right)_{\lambda a}$ $\times \psi_{a}$, where $\theta$ is the vacuum mixing angle. We suggest that the mass eigenstates are Dirac particles. In the basis of the mass eigenstates $\psi_{a}$ neutrinos have definite masses $m_{a}$.

In the limit of small neutrino masses the wave equations for the upper $\xi_{a}$ and lower $\eta_{a}$ chiral components of the spinor $\psi_{a}^{\mathrm{T}}=\left(\xi_{a}, \eta_{a}\right)$ decouple. Therefore

${ }^{a}$ e-mail: maxim.dvornikov@usm.cl 
using cylindrical coordinates $(r, \phi, z)$ with $\boldsymbol{\Omega}=\Omega \mathbf{e}_{z}$ we can write the general expression for the two component wave function $\eta_{a}$ in the form (see Ref. [2]),

$$
\begin{array}{r}
\eta_{a}(r, \phi, t)=\sum_{\mathrm{n}, \mathrm{s}=0}^{\infty}\left(a_{\mathrm{n} s}^{(a)}(t) u_{a, \mathrm{n} s}^{+}(r, \phi) \exp \left[-\mathrm{i} E_{\mathrm{n}}^{(a)+} t\right]\right. \\
\left.+b_{\mathrm{n} s}^{(a)}(t) u_{a, \mathrm{n} s}^{-}(r, \phi) \exp \left[-\mathrm{i} E_{\mathrm{n}}^{(a)} t\right]\right),
\end{array}
$$

where the energy levels

$$
E_{\mathrm{n}}^{(a) \pm}=-V_{a} \pm \sqrt{4 V_{a} \Omega \mathrm{n}+m_{a}^{2}}, \quad \mathrm{n}=0,1,2, \ldots
$$

have the discrete values and the basis spinors

$$
u_{a, \mathrm{n} s}^{( \pm)}(r, \phi)=\sqrt{\frac{V_{a} \Omega}{2 \pi}}\left(\begin{array}{c}
I_{\mathrm{n}-1, s}\left(\rho_{a}\right) e^{\mathrm{i}(l-1) \phi} \\
\mp \mathrm{i} I_{\mathrm{n}, s}\left(\rho_{a}\right) e^{\mathrm{i} l \phi}
\end{array}\right), \quad l=\mathrm{n}-s,
$$

are expressed in terms of the Laguerre functions $I_{\mathrm{n}, s}\left(\rho_{a}\right)$ of the dimensionless argument $\rho_{a}=V_{a} \Omega r^{2}, V_{1}=G_{\mathrm{F}}\left(n_{n}-2 n_{e} \sin ^{2} \theta\right) / \sqrt{2}$ and $V_{2}=G_{\mathrm{F}}\left(n_{n}-\right.$ $\left.2 n_{e} \cos ^{2} \theta\right) / \sqrt{2}$ are the potentials of the interaction of mass eigenstates with background matter. Note that in Eqs. (3)-(5) we study neutrinos propagating in the equatorial plane with $z=0$.

In Ref. [2] we obtained the general differential equations for the coefficients $a_{\mathrm{n} s}^{(a)}(t)$ and discussed the situation of the small initial angular momentum: $l \ll s$. Now we study neutrino flavor oscillations for $l \gg s$, i.e. particles with big initial angular momentum. Using the results of our work [2] we get that in this situation the differential equations for different $l$ and $s$ decouple and we can describe the evolution of the system with help of the single Schrödinger equation,

$$
\mathrm{i} \frac{\mathrm{d}}{\mathrm{d} t}\left(\begin{array}{c}
\tilde{a}_{l}^{(1)} \\
\tilde{a}_{l}^{(2)}
\end{array}\right)=\left(\begin{array}{cc}
\omega / 2 & \Delta \\
\Delta & -\omega / 2
\end{array}\right)\left(\begin{array}{c}
\tilde{a}_{l}^{(1)} \\
\tilde{a}_{l}^{(2)}
\end{array}\right),
$$

where the components $\tilde{a}_{l}^{(a)}$ of the "wave function", which now can be enumerated with the single quantum number "l" are related to the coefficients in Eq. (3) by the formula, $\tilde{a}_{l}^{(a)}=\left(\exp \left[-\mathrm{i} \sigma_{3} \omega / 2\right]\right)_{b}^{a} a_{l}^{(b)}$. The parameters of the effective Hamiltonian in Eq. (6) have the form,

$$
\Delta=\left(\frac{G_{\mathrm{F}}}{\sqrt{2}}-\frac{k}{2 n_{n}}\right) n_{e} \sin 2 \theta, \quad \omega=\frac{\delta m^{2}}{2 k}, \quad k=\sqrt{4 V \Omega l},
$$

where $k$ is the effective momentum of neutrinos and $V=G_{\mathrm{F}} n_{n} / \sqrt{2}$.

Using Eqs. (6) and (7) we can obtain the transition probability in the form,

$$
P_{\beta \rightarrow \alpha}(x)=\frac{(\Delta \cos 2 \theta+\omega \sin 2 \theta / 2)^{2}}{\Delta^{2}+(\omega / 2)^{2}} \sin ^{2}\left(\sqrt{\Delta^{2}+(\omega / 2)^{2}} x\right) .
$$




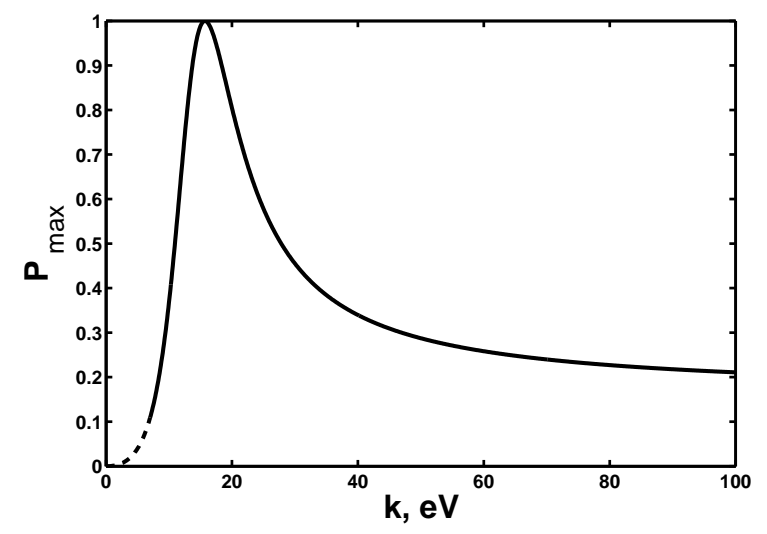

Figure 1: The dependence of the maximal transition probability on the neutrino energy for the case $l \gg s$. This plot corresponds to $\nu_{e} \rightarrow \nu_{\mu}$ oscillations, with $\delta m^{2} \approx 8.1 \times 10^{-5} \mathrm{eV}^{2}$ and $\sin ^{2} \theta \approx 0.3$ and matter with $n_{n}=10^{38} \mathrm{~cm}^{-3}$ and $Y_{e}=n_{e} / n_{n}=3 \times 10^{-3}$. The very low energy part of the curve, which cannot be treated in frames of the quantum mechanical approach, is shown by the dashed line.

Let us discuss the oscillation scheme $\nu_{e} \rightarrow \nu_{\mu}$. In Fig. 1 we present the maximal transition probability as a function of the neutrino energy, built on the basis of Eq. (8). Note that for the nuclear matter in $\beta$ equilibrium the number density of electrons has the following value: $n_{e} \approx 3 \pi^{2} n_{n}^{2} /\left(2 m_{n}\right)^{3}$. As one can see from this picture, the transition probability has a resonance behaviour. The maximal transition probability is reached at $\sim 16 \mathrm{eV}$. At very large energies the transition probability approaches to the limit $P_{\max } \rightarrow \cos ^{2}(2 \theta) \approx 0.16$, a result that can also be inferred from Eq. (8). We should notice that the solution presented in Eq. (8) is not valid for very large neutrino energies, because in that case the condition $l \gg s$ is violated.

\section{Acknowledgments}

The work has been supported by Conicyt (Chile), Programa Bicentenario PSD91-2006. The author is thankful to C. O. Dib and for helpful discussions and the organizers of $14^{\text {th }}$ Lomonosov conference for the invitation.

\section{References}

[1] A. V. Grigoriev, et al., Russ. Phys. J. 50, 845 (2007);

A. I. Studenikin, J. Phys. A 41, 164047 (2008).

[2] M. Dvornikov and C. O. Dib, 0907.1445 [astro-ph]; M. Dvornikov, to be published in Appl. Comput. Math., 1001.2516 [hep-ph]. 